\title{
EDITORIAL
}

\section{A Futuristic Vision of Forensic Science}

\author{
Itiel E. Dror, ${ }^{1,2}$ Ph.D.; and Ruth M. Morgan , ${ }^{1,2}$ D.Phil. \\ ${ }^{1}$ UCL Centre for the Forensic Sciences, University College London, London, U.K. \\ ${ }^{2}$ UCL Department of Security and Crime Science, University College London, London, U.K.
}

What will forensic science look like in the future? Forensic science has a promising future as new methods, technologies, and scientific advancements create new possibilities that have not yet been imagined. For example, while the discovery and utilization of DNA has transformed current-day forensic science, it will continue to do so as the detection technologies develop and our understanding of trace DNA transfer, persistence, prevalence, and recovery grows. New discoveries can create incremental evolutionary changes or revolutionary changes that will reshape the face of forensic science all together. We cannot predict the innovations and new technologies that will come to be, but we can certainly expect that they will happen (1) and that they will create new possibilities for forensic science.

In this piece, we engage in a thought exercise to consider and present a view of what forensic science may look like in the future. It is intended to share ideas and provoke discussions about the direction forensic science may take in the future, what it can be, and what it will be capable of. This futuristic view takes into account that one of the most critical, challenging, and foundational aspects of forensic science is that it is a complex interdisciplinary field, with multiple stakeholders, drivers, and pressures (2). For this reason, the potential of new technologies and an increased evidence base to underpin crime reconstructions is clear. While we present here a vision of how these new capabilities may shape forensic science in the future, it is beyond the scope of this piece to provide technical details of how these developments may be implemented given that many of these technologies have yet to be created.

Forensic science is often driven by specific issues and scandals (such as a miscarriage of justice), and resources are specifically deployed to address them (3). This means that forensic science is often reactive to "symptoms" that arise $(4,5)$, rather than engaging in continuous and systematic proactive examination, research, and self-reflection as routine practice. In addition, forensic science frequently works within the framework that "every case is different," which creates a fundamental tension between research seeking to develop generalizable theories and approaches, and professional practices in crime reconstruction. Therefore, taking a longer term view of the possibilities and potentially desirable directions of the future of forensic science is an important undertaking.

As we consider a vision of the future, one of the basic challenges is that some forensic domains have developed within investigative practices (e.g., Ref. [6]) rather than first establishing the principles and foundations of a domain through scientific research. This has also led to many forensic domains having (comparatively) small amounts of data (e.g., Ref. [7]). Courts have readily accepted forensic science evidence (8), and given the complex ecosystem of forensic science with many interacting variables (9), the courts have allowed forensic science to flourish even without a truly holistic and coherent overview of crime reconstruction approaches, and a scientific research culture (10). As we look forward, one thing is almost certain, in the future datasets will be larger, our use and reliance on technology and laboratory information management systems will grow, and it will be possible to ensure that decision making is more transparent, which will transform narrow and anecdotal approaches.

In this thought exercise to envision the future, timescale is a critical variable, because the further into the future we go, the more unknown and unpredictable factors there will be. However, it is possible to predict that forensic science in the future will take advantage of the development and utilization of emerging technologies, which will create new capacities to capture, produce, store, search, synthesize, visualize, and interrogate data.

It is also clear that with all these new and exciting possibilities, new challenges and vulnerabilities also arise. Utopian fiction highlights the potential for this kind of data-rich world to transform society and human nature, but these new technologies and capabilities also create a world that faces very serious challenges (such as disclosure [11] and issues around ethics and privacy $[12,13]$ ), and there will certainly be new (as yet unknown) challenges. 
Taking a horizon scanning approach in considering forensic science in the future, it is important to consider how these new capabilities from technological advances and the ability to capture and process data might transform forensic science, and how this coming revolution can change and impact forensic science. Regardless of the specific details of these advances (some of which will make huge impacts, such as that made by DNA), it is possible to anticipate that future technologies will enable the creation of a platform that will manage and integrate forensic work within a simulator system that could be considered to be an "Integrative Reconstruction and Prediction Simulator" (IRPS). Such a unified platform will make it possible to integrate findings from a very broad range of physical and digital materials, situated within all the relevant contextual information in order to scientifically reconstruct a crime event, in a way that takes a truly holistic integrative approach to forensic science (14-16).

In this thought exercise of taking a futuristic view of forensic science, it can be anticipated that it will be possible to simulate and model different scenarios and outcomes, similar to some extent to those in use in the domains of aviation and medicine (e.g., Ref. [17-19]). However, rather than having the human body as the object of inquiry-as in medical simulations - the IRPS will have the crime scene as its object of inquiry. It will be possible to run multiple simulations utilizing all the different forms of forensic science evidence and incorporating the context of each piece of intelligence and evidence. In so doing, it will be possible to provide statistical probabilities for different scenarios that can inform a reconstruction of what may have happened, by whom, and when, and potentially contribute to crime prevention tools and approaches (20).

By running a multitude of different possible reconstructions of a crime, this kind of platform would enable an assessment of the possibilities of different scenarios, providing a differential crime diagnosis, to inform the likelihoods of what could have happened given the forensic science evidence available (in combination with all other forms of intelligence that may exist). This approach of differential diagnosis is already being realized in the medical domain where it is possible to distinguish between different conditions, which can give rise to similar signs or symptoms (e.g., Ref. [21,22]). In a system where it is possible to incorporate all the information and evidentiary materials relevant to a case into an IRPS, it can be anticipated that the IRPS will be able to only use information that is task-relevant for each piece of forensic science evidence (23). Then, using context management tools, such as Linear Sequential Unmasking (LSU), the IRPS could optimize the sequence of examining and interpreting the evidence, making sure the evidence is driving the crime reconstruction process, rather than a target suspect (24).

A tool of this kind in this futuristic vision of forensic science will be able to take into account not only the analysis of the forensic science evidence, but, when scientifically relevant, it will also take into account where it was found in the crime scene, what it was associated with, and a whole array of relevant contextual information, as appropriate. For example, the locations and orientations of latent fingermarks can be combined and integrated with locations and orientations of patterns of blood spatter whereby they constitute relevant context to each other, so the likelihood of multiple different crime reconstructions can be simulated by the IRPS by running various scenarios given all the crime scene evidence, utilizing Bayesian and other statistical tools (e.g., Ref. [16,25]).

The potential of a tool in the future such as the IRPS will be that it may not only provide indications of the likelihood of the source attribution, but it will also be able to draw conclusions at activity and offense levels $(14,16,26)$. By running multiple simulations that consider different scenarios as to what happened, by whom, and when, it will be able to distinguish, for example, between primary and secondary transfer of trace DNA (27), and to give information, such as a facial image, of who committed a crime with a measure of likelihood on the basis of the simulation data produced. However, when the forensic science evidence is not conclusive and a number of possible scenarios are viable, then non-scientific lines of evidence (such as eye witness testimony) could be used to further assess the different scenarios, in a way that ensures transparency in terms of what intelligence/evidence was used in computing each possible scenario, which will be routinely recorded and documented.

With such future capabilities, forensic science may not only utilize the IRPS to reconstruct and solve crimes, but this kind of IRPS tool will ensure greater transparency in the documentation of how reconstructions were computed during the original analysis. Hence, it will enable better communication and documentation of the decision pathways that resulted in the findings and establishment of the weight of the evidence provided to the fact finders. For example, it could be possible that using virtual reality (VR) approaches, the IRPS would enable a fully transparent presentation of the different possible alternative crime reconstructions, and the reasons for their likelihood, including any uncertainties, limitations, biases, and assumptions.

Of course, the creation of this kind of capability will change the role of the human examiner, their use and reliance on technology, and how work is distributed between human and machine (28). These will have far-reaching implications to selection, training, experiences, and competencies of the human examiner (29). The value of forensic science with a tool with this kind of capability would be its ability to create and utilize large datasets through simulations of different scenarios that incorporate multiple variables. In so doing, it will be able to consider the complex ecosystem of forensic science and therefore identify potential root causes of specific challenges that currently exist and that will arise through the creation of new technological capabilities. It will bring together both experimental data and professional practitioner experience, and it will ensure the means of documenting every stage and decision so that 
cases can be re-evaluated in the future if new information or technologies become available. To ensure integrity of the IRPS, it will be important to preserve its transparency and so it will need to be open source, and freely available and enable to offer open forensic science (30), with full legal and forensic disclosures (31).

Our futuristic vision of forensic science with the developing capabilities of simulation and predictions of tools, such as an IRPS, will also have a role to play before crimes occur. First, the IRPS will be able to contribute to providing intelligence for the disruption and prevention of existing crime types (32). For example, it may well be possible to not only deal with a single crime scene, but also create a database within the IRPS of all other crimes, past and present, which will then enable connections and inferences across crime events to be made, regularities and common themes to be found, and intelligence that can be deployed to disrupt and prevent crime produced.

Second, as society and technological capabilities change, existing crime types will need new forensic tools $(20,33)$. For example, already we are seeing that changes in paper currency to polymer require new techniques for the development of fingermarks deposited on the new plastic notes. As it becomes possible to capture, retain, store, and search greater amounts of data, a system such as the IRPS would have the capacity to store and use a whole range of data, such as the chemicals used in the manufacture of paint and ink, the patterns of different tire treads, and the digital signatures of various devices to enhance evaluative interpretation. The IRPS may also be able to record and make use of artificially modified elements in the production of goods (such as firearms) so they can be easily traced and identified if used in a crime.

Third, a further capability of future IRPS forensic science tools will be in identifying and anticipating new forms of crime that are enabled by emerging technologies, such as new digital capabilities, AI, machine learning, remote sensing, robotics, electronic remote control, autonomous vehicles, and drones. These will inevitably and undoubtedly be beneficial to society, but they will also present opportunities for new types of crime.

Anticipating these future crimes by using the capacity of an IRPS system will enable a proactive approach for forensic science to not only detect but also predict. Through the anticipation of future crimes, it is possible to engineer systems to increase the risk that the criminals will be caught, or reduce the reward of committing the crime, thereby contributing to crime prevention approaches (34). Hence, forensic science in the future will not only focus on crime reconstructions and interpreting of forensic science evidence from a crime scene, but will also have the capability to "out think crime" and create a more proactive forensic science.

It is not possible to predict the specific details of technological and scientific discoveries that will shape and advance forensic science in the future (nor the practical details of the "nuts \& bolts" of how these will be implemented), but it is possible to be confident that advances in technology and science will happen. However, to realize the potential of these new discoveries, we need to engage in thought exercises so as to develop a vision of the solution that is currently needed that new capabilities may be able to achieve. Enhanced capabilities of data management, simulation, and data creation that can address complexity and the dynamic nature of forensic science reconstructions in individual cases could address the deep and systemic challenges we face in crime reconstructions and interpreting forensic science evidence (4).

Therefore, it is possible to envision in this thought exercise a technological platform that will revolutionize the practice of forensic science. Such a platform offers the ability to incorporate existing forensic tools within new capabilities. It will go beyond source attribution to activity and offense levels, and will incorporate virtual reality and immersive technologies to assist investigators and the courts (35). It will also offer the means to ensure real transparency in terms of the variables considered and the decisions made that can be preserved for re-examinations if needed. The future certainly looks bright, but given that complex challenges require collaborative and holistic solutions, to achieve this kind of capability will require a truly interdisciplinary approach from across the sciences, arts, and humanities that brings these emerging capabilities together with experts from computer science, statistics, cognitive science, and other related domains. In addition, we need to ensure that we focus on both technological capabilities and foundational research that underpins evaluative interpretation $(5,36)$. Such a forensic science is an exciting possibility we can look forward to.

\section{References}

1. Taleb NN. The black swan: the impact of the highly improbable. New York, NY: Random House is the Publisher, 2007.

2. Morgan RM. Conceptualising forensic science and forensic reconstruction. Part II: the critical interaction between research, policy/law and practice. Sci Justice 2017;57(6):460-7.

3. Cole SA. Scandal, fraud, and the reform of forensic science: the case of fingerprint analysis. W Va L Rev 2016;119(2):52448.

4. Morgan RM. Forensic science. The importance of identity in theory and practice. Forensic Sci Int Synergy 2019;1:239-42.

5. Morgan RM, Levin E. A crisis for the future of forensic science: lesson from the UK for the importance of epistemology for funding research and development. Forensic Sci Int Synergy 2019;1:243-52.

6. Garfinkel SL. Digital forensics research: the next 10 years. Digit Investig 2010;7(Suppl):S64-73. 
7. Gosch A, Courtslow C. On DNA transfer: the lack and difficulty of systematic research and how to do it better. Forensic Sci Int Genetics 2019;40:24-36.

8. The Law Commission. Expert evidence in criminal proceedings in England and Wales. The Forty-Fifth Annual Report of the Law Commission. London, U.K.: The Stationery Office Limited, 2011.

9. Roux C, Crispino F, Ribaux O. From forensics to forensic science. Current Issues Crim Just 2012;24(1):7-24.

10. 1Mnookin J, Cole S, Dror IE, Fisher B, Houck M, Inman K, et al. The need for a research culture in the forensic sciences. UCLA Law Rev 2011;58(3):725-79.

11. McCartney C. Commentary: disclosure in the criminal justice system. J Forensic Legal Med 2018;58:72-3.

12. House of Commons Science and Technology Committee. The work of the Biometrics Commissioner and the Forensic Science Regulator. Nineteenth Report of Session 2017-2019. 2019https://publications.parliament. uk/pa/cm201719/cmselect/cmsctech/1970/1970.pdf(accessed September 10, 2019).

13. Murphy E. Law and policy oversight of familial searches in recreational genealogy databases. Forensic Sci Int 2018;292:e5-e9.

14. Morgan RM. Conceptualising forensic science and forensic reconstruction. Part I: a conceptual model. Sci Justice 2017;57(6):455-9.

15. Morgan RM, Nakhaeizadeh S, Earwaker H, Rando C, Harris AJL, Dror IE. Interpretation of evidence: cognitive decision making under uncertainty (at every step of the forensic science process). In: Wortley R, Sidebottom A, Laycock G, Tilley N, editors. Handbook of crime science. Abingdon, U.K.: Routledge, 2018;408-20.

16. Koeijer J, Sjerps M, Vergeer P, Berger C. Combining evidence in complex cases - a practical approach to interdisciplinary casework. Sci Justice 2019. https://doi.org/10.1016/j.scijus.2019.09.001.

17. Lee TE. Flight simulation. London, U.K.: Routledge, 2005.

18. Kaufman JJ, Luo G, Siffert RS. Ultrasound simulation in bone. IEEE Trans Ultrason Ferroelectr Freq Control 2008;55(6):1205-18.

19. Kunkler K. The role of medical simulation: an overview. Int J Med Robot 2006;2(3):203-10.

20. Dror IE. Cognitive neuroscience in forensic science: understanding and utilising the human element. Philos Trans R Soc Lond B Biol Sci 2015;370(1674):20140255.

21. Maudea J. Differential diagnosis: the key to reducing diagnosis error, measuring diagnosis and a mechanism to reduce healthcare costs. Diagnosis 2014;1(1):107-9.

22. Barondess JA, Carpenter CC, editors. Differential diagnosis. Philadelphia, PA: Lea \& Febiger, 1994.

23. Dror IE. Biases in forensic experts. Science 2018;360(6386):243.

24. Dror IE, Thompson WC, Meissner CA, Kornfield I, Krane D, Saks M, et al. Context management toolbox: a Linear Sequential Unmasking (LSU) approach for minimizing cognitive bias in forensic decision making. J Forensic Sci 2015;60(4):1111-2.

25. Neumann C, Evett IW, Skerrett J. Quantifying the weight of evidence from a forensic fingerprint comparison: a new paradigm. J R Stat Soc Ser A Stat Soc 2012;175(2):1-26.

26. Cook R, Evett IW, Jackson G, Jones PJ, Lambert JA. A hierarchy of propositions: deciding which level to address in casework. Sci Justice 1998;38(4):231-9.

27. Taylor D, Biedermann A, Samie L, Pun KM, Hicks T, Champod C. Helping to distinguish primary from secondary transfer events for trace DNA. Forensic Sci Int Genet 2017;28:155-77.

28. Dror IE, Mnookin J. The use of technology in human expert domains: challenges and risks arising from the use of automated fingerprint identification systems in forensics. Law Probab Risk 2010;9(1):47-67.

29. Dror IE. What is (or will be) happening to the cognitive abilities of forensic experts in the new technological age. J Forensic Sci 2013;58 (2):563.

30. Chin JM, Ribeiro G, Rairden A. Open forensic science*. J Law Biosci 2019;6(1):255-288.

31. Almazrouei MA, Dror IE, Morgan RM. The forensic disclosure model: what should be disclosed to, and by, forensic experts? Int J Law Crime Justice 2019;100330. https://doi.org/10.1016/j.ijlcj.2019.05.003.

32. Ribaux O, Talbot Wright B. Expanding forensic science through forensic intelligence. Sci Justice 2014;54(6):494-501.

33. Smolianitsky E, Wolf E, Almog J. Proactive forensic science: a novel class of cathinone precursors. Forensic Sci Int 2014;242:219-27.

34. Clarke RV. Technology, criminology and crime science. Eur J Crim Pol Res 2004;10(1):55-63.

35. Gelder J, Vries RE, Demetriou A, Sintemaartensdijk I, Donker T. The virtual reality scenario method: moving from imagination to immersion in criminal decision-making research. J Res Crime Delinq 2019;56 (3):451-80.

36. House of Lords Science and Technology Select Committee. Forensic science and the criminal justice system: a blueprint for change. 3rd Report of Session 2017-2019. HL Paper 333. 2019.

https://publications.parliament.uk/pa/ld201719/ldselect/ldsctech/333/333.pdf (accessed September 13, 2019). 